\title{
One-Year Outcomes of Women Started on Antiretroviral Therapy during Pregnancy before and after the Implementation of Option B+ in Malawi: A Retrospective Chart Review from Three Facilities
}

\author{
Alfred A. Kamuyango 1*, Lisa R. Hirschhorn², Wenjia Wang', Perry Jansen", \\ Risa M. Hoffman 5 \\ ${ }^{1}$ Department of Pathology, College of Medicine, University of Malawi, Blantyre, Malawi \\ ${ }^{2}$ Department of Global Health and Social Medicine, Harvard Medical School, \\ Boston, USA \\ ${ }^{3}$ Department of Statistics, University of California, Los Angeles, USA \\ ${ }^{4}$ Hope Medical Centre, Lilongwe, Malawi \\ ${ }^{5}$ Department of Medicine and Division of Infectious Diseases, David Geffen School of Medicine, \\ University of California, Los Angeles, USA \\ Email: akamuyango@medcol.mw
}

Received 20 June 2014; revised 15 July 2014; accepted 10 August 2014

Copyright (C) 2014 by authors and Scientific Research Publishing Inc.

This work is licensed under the Creative Commons Attribution International License (CC BY).

http://creativecommons.org/licenses/by/4.0/

(c) (i) Open Access

\section{Abstract}

Objective: To compare one-year outcomes of women started on antiretroviral therapy (ART) during pregnancy in the pre-Option $B+$ era to those in the Option $B+$ era. Methods: A retrospective chart review was performed at three sites in Malawi. Women were included in the "pre-Option $\mathrm{B}+$ " cohort if they started ART during pregnancy for a CD4 count $<350$ cells $/ \mathrm{mm}^{3}$ or WHO $3 / 4$ condition and in the "Option B+" cohort if they started ART during pregnancy regardless of CD4 count or clinical stage. One-year outcomes were compared using Fisher's exact and ANOVA F-tests. Results: A higher proportion of women in the pre-Option B+ cohort started ART at WHO stage 3/4 (11.9\% versus $1.1 \%, P<0.001)$, switched ART regimens $(5.9 \%$ versus $0 \%, P=0.002)$, or died in the first year after starting treatment $(3.9 \%$ versus $0.5 \%, P=0.05)$. While more women in the Option $B+$ cohort had poor adherence or defaulted, these differences were not significant. Conclusions: At our study sites, the transition to Option B+ has been associated with ART initiation in women with less advanced HIV infection, improved medication tolerability, and lower mortality. Further re-

\footnotetext{
"Corresponding author.
}

How to cite this paper: Kamuyango, A.A., et al. (2014) One-Year Outcomes of Women Started on Antiretroviral Therapy during Pregnancy before and after the Implementation of Option B+ in Malawi: A Retrospective Chart Review from Three Facilities. World Journal of AIDS, 4, 332-337. http://dx.doi.org/10.4236/wja.2014.43039 
search is needed to better understand outcomes of Option B+.

Keywords

Antiretroviral Therapy, Pregnancy, Option B+, Prevention of Mother-to-Child Transmission

\section{Introduction}

Prevention of mother-to-child transmission (PMTCT) guidelines in resource-limited settings has been evolving over time, with the 2013 WHO Guidelines recommending either Option B, antiretroviral therapy (ART) during pregnancy and breastfeeding for women who do not meet criteria for lifelong ART by CD4 count or clinical stage, or Option B+, lifelong ART for all pregnant and breastfeeding women regardless of CD4 count or clinical stage [1]. In 2011, Malawi became one of the first countries in the world to adopt Option B+ as part of their national HIV policy. This decision was made prior to the WHO 2013 guidelines and was a response to low uptake of PMTCT in Malawi (39\%) [2], high fertility rates resulting in an average of 5.7 births per woman [3], slow progress toward 2015 Millennium Development Goals in maternal and child health, and proven efficacy of three-drug ART in lowering MTCT rates to $<2 \%$ [4].

Given Malawi's position as one of the earliest implementers of Option B+, it is also one of the first countries to have early data on outcomes. The Malawi Ministry of Health (MOH) quarterly report has placed 12-month Option B+ retention at 77\% [5] and recently published nationwide data showed that most losses occur within three months of ART initiation [6]. In this nationwide sample, pregnant women were five times as likely to be lost to follow-up compared to non-pregnant women initiating therapy for disease stage or CD4 count $<350$ cells $/ \mathrm{mm}^{3}$. To date, with the exception of retention data, there are no published studies of other outcomes of women in Option B+ in Malawi. The objective of this study was to perform a retrospective chart review to compare maternal outcomes one year after starting ART in pregnancy in the pre-Option B+ era to outcomes in the Option B+ era. We hypothesized that women enrolled in the Option B+ era would have higher CD4 counts and lower clinical stage prior to ART, but would be more likely to have low adherence and more likely to default from care.

\section{Methods}

\subsection{Study Setting, Inclusion Criteria, and Sampling}

We performed a retrospective cohort study using routine data captured on standardised Malawi Ministry of Health (MOH) patient charts (ART mastercards) and $\mathrm{MOH}$ patient tracking logs (ART registers). The study was conducted at three ART sites in central and southern Malawi. In southern Malawi study sites included Malamulo Hospital, a faith-based facility in which antenatal, maternity, and HIV care are delivered, and Thyolo District Hospital, a public health facility also providing care for antenatal, maternity, and HIV patients. Both of these facilities provide free HIV care. The third site, Partners in Hope Medical Center, is a faith-based facility in central Malawi that does not provide antenatal or maternity care, but offers free HIV care, including postpartum followup for women started on ART during pregnancy.

Women were included in the pre-Option B+ cohort if they were started on ART during pregnancy prior to July 2011, per guidelines at that time, which included initiation for a CD4 count $<350 \mathrm{cells} / \mathrm{mm}^{3}$ or a World Health Organization (WHO) stage 3 or 4 clinical condition. The sampling timeframe for the pre-Option B+ cohort was initiation of ART between 1 September 2008-1 September 2010. Women who had a CD4 count $\geq 350$ cells/ $/ \mathrm{mm}^{3}$ and received single or dual short course antiretrovirals for PMTCT were excluded from this cohort. Women were included in the Option B+ cohort if they started ART during pregnancy after July 2011 under the new Option $\mathrm{B}+$ treatment guidelines, regardless of CD4 count or clinical stage. The sampling timeframe for the Option B+ cohort was ART initiation between 1 September 2011-30 April 2012. For both the pre- and Option B+ cohorts, pregnant women were included if they had transferred into the clinic if they met inclusion criteria, regardless of where they were initiated on ART. We used systematic sampling by physically selecting every third mastercard of the eligible women for each cohort included in the study. 


\subsection{Data Collection and Statistical Analyses}

Baseline information on patients was abstracted from available data on standardized $\mathrm{MOH}$ mastercards, including age at ART initiation, WHO clinical stage at ART initiation, baseline CD4 cell count (where available), presence of tuberculosis (TB) and/or Kaposi's Sarcoma (KS) at baseline, date of ART initiation, and ART regimen at initiation. We abstracted the following treatment outcomes one year after ART initiation for all women: 1) alive and on ART; 2) died for any reason; 3) defaulted (defined in the national guidelines as not seen in the ART clinic and off ART for more than 60 days, and not known to have died or transferred out). Other treatment outcomes recorded included less than 95\% adherence (as documented in the mastercard) at either of the last 2 visits during the one-year follow-up period, and switching (one or more medication changes) of ART to another regimen due to toxicity.

We used Fisher's exact tests for categorical variables and ANOVA F-tests for continuous variables to compare demographics and outcomes between the two cohorts. Statistical analyses were performed using R Software (Foundation for Statistical Computing, Vienna, Austria). The significance level was set at $\mathrm{P} \leq 0.05$. The study was approved by the Malawi National Health Sciences Research Committee (Protocol \# 963) and given a non-human subject designation by the University of California, Los Angeles Internal Review Board.

\section{Results}

\subsection{Baseline Characteristics of Pre-Option B+ and Option B+ Cohorts}

A total of 102 women were included in the pre-Option B+ cohort and 190 in the Option B+ cohort. Baseline patient characteristics are summarised in Table 1 . The median age in the pre-Option $\mathrm{B}+$ cohort was slightly older at 29 years (interquartile range (IQR): 25 - 32) compared to 27 years (IQR: 24 - 31) in the Option B+ cohort (P $=0.002)$. Among women with a CD4 count documented $(\mathrm{N}=108)$, those in the pre-Option $\mathrm{B}+$ cohort had a lower median CD4 cell count compared with women in the Option B+ group (231 cells/ $\mathrm{mm}^{3}$ versus 558 cells/ $\mathrm{mm}^{3}, \mathrm{P}<0.001$ ). A higher proportion of women in the pre-Option B+ cohort were WHO stage 3 or 4 at the time of ART initiation $(11.9 \%$ versus $1.1 \%, \mathrm{P}<0.001)$. Of the clinical conditions captured on the ART mastercard (Tuberculosis and Kaposi's sarcoma), Kaposi's sarcoma at ART initiation was more frequent in the pre-Option $\mathrm{B}+$ cohort $(2.9 \%$ versus $0 \%, \mathrm{P}=0.04)$. All patients in the pre-option $\mathrm{B}+$ cohort were started on a first-line regimen of stavudine (d4T), lamivudine (3TC), and nevirapine (NVP) per country guidelines at that time, whereas all patients in the Option B+ cohort were started on a first line regimen of tenofovir (TDF), lamivudine (3TC), efavirenz (EFV).

Table 1. Baseline characteristics of patients starting ART in the pre-Option B+ cohort compared to women starting ART in the Option B+ cohort.

\begin{tabular}{cccc}
\hline Characteristics & Pre-Option B+ Cohort (N = 102) & Option B+ Cohort (N = 190) & P-value \\
\hline Mean age (IQR) & $29(25-32)$ & $27(24-31)$ & 0.002 \\
Median baseline CD4 count (IQR) & $231(165-269)$ & $558(493-700)$ & $<0.001$ \\
WHO clinical stage* & & $188(99.0)$ & $<0.001$ \\
Stage 1 or 2, N (\%) & $89(88.1)$ & $2(1.1)$ & $<0.001$ \\
Stage 3 or 4, N (\%) & $12(11.9)$ & 0 & 0.04 \\
Opportunistic infection at ART initiation & & 0 & 0.35 \\
Kaposi’s sarcoma, N (\%) & $3(2.9)$ & $1(1.0)$ & 0 \\
Active tuberculosis, N (\%)
\end{tabular}

IQR: Interquartile range; *Stage missing for one woman in the pre-Option B+ cohort; ^Available for 95 women in the pre-Option B+ cohort and 13 in the Option B+ cohort.

\subsection{One-Year Outcomes of Pre-Option B+ and Option B+ Cohorts}

One-year outcomes by cohort are summarised in Table 2. In the pre-Option B+ cohort, four women died (3.9\%); 
one defaulted (0.9\%), and two (2.0\%) had incomplete treatment adherence. Six women (5.9\%) switched their ART regimen due to toxicity ( 5 stopped NVP for hepatitis and/or rash and 1 stopped d4T for neuropathy). In the Option B+ cohort, there was one death (0.5\%), five women (2.6\%) defaulted, and eight (4.2\%) had inadequate treatment adherence. No women switched ART regimens. There was a higher proportion of deaths and switching of ART regimens in the pre-Option $\mathrm{B}+$ cohort $(3.9 \%$ versus $0.05 \%, \mathrm{P}=0.05 \%$ and $5.9 \%$ versus $0 \%, \mathrm{P}=$ 0.002 , respectively). While default and incomplete adherence were more common in the Option B+ cohort, these differences were not statistically significant. We analysed the combined endpoint of default and death and did not find statistically significant differences between the pre-Option B+ and B+ cohorts.

Table 2. One-year outcomes of women on antiretroviral therapy in the pre-Option B+ cohort versus the Option B+ cohort.

\begin{tabular}{cccc}
\hline Outcome one-year after starting ART in pregnancy & Pre-Option B+ (n = 102) & Option B+ (n = 190) & P-value \\
\hline Default", N (\%) & $1(1.0)$ & $5(2.6)$ & 0.67 \\
Death, N (\%) & $4(3.9)$ & $1(0.5)$ & 0.05 \\
Less than 95\% adherence^, N (\%) & $2(2.0)$ & $8(4.2)$ & 0.50 \\
ART switching due to toxicity ( $\geq 1$ switch), N (\%) & $6(5.9)$ & 0 & 0.002 \\
\hline
\end{tabular}

*Lost from care and out of ART for 60 or more days and not known to have died or transferred out; ${ }^{\wedge}$ Categorized as <95\% adherence based on pill counts from the last two visits during the one year follow-up period (as documented in the ART mastercard).

\section{Discussion}

As expected under the new guidelines, women starting ART in the Option B+ era had fewer WHO 3/4 conditions, higher CD4 cell counts (among those measured) and lower mortality. While more women in the Option B+ cohort had poor adherence or default, these differences were not statistically significant, possibly due to small numbers in our sample and resultant low power. Overall, there were very low rates of default one year after starting ART in both pre- and Option B+ cohorts; however, there is an emerging body of data about the challenges of adherence and retention in Option B+. The Malawi Ministry of Health quarterly report data has shown $23 \%$ of patients are not retained at 12 months [5]. A program in Malawi reported 20.4\% of women were lost within 3 months of ART initiation [7] and this report has been followed by more recent nationwide data from Malawi showing that $17 \%$ of women in Option B+ are lost to follow-up 6 months after ART initiation, with most lost within three months [6]. In this study, pregnant women were five times as likely to be lost to follow-up compared to non-pregnant women initiating therapy for disease stage or CD4 count $<350$ cells $/ \mathrm{mm}^{3}$ and were also more likely to never return to clinic after their initial visit (OR 5.0, 95\% CI: 4.2 - 6.1). Yet another study suggested that ART adherence in women on Option B+ decreases with older age (27 and older) and shorter time on ART (less than 3 months) [8].

Pregnant and postpartum women with HIV have been known to have challenges with adherence and retention, irrespective of PMTCT approach. Several studies from Africa have shown that pregnant and postpartum women have high rates of loss to follow-up [9] [10], including one study that reported a hazard of loss to follow up for pregnant women after 6 months of ART of 3.75 (95\% CI 1.53-9.16) compared to men [11]. Additionally, despite being retained in care, adherence is a challenge, with a meta-analysis of studies from the US and 4 countries in Africa suggesting that only $73.5 \%$ of pregnant women achieve optimal adherence, with adherence remaining suboptimal in the postpartum period [12]. Our data showed better tolerability of the Option B+ regimen (3TC, TDF, EFV) with no ART switches compared to the pre-Option B+ regimen (d4T, 3TC, NVP), which required switches in $5.9 \%$ of women. Studies have not yet shown whether improved tolerability of modern ART regimens is associated with improved postpartum adherence and retention.

There are several possible challenges to adherence and retention in Option B+ programs. Healthier women may not see the need to continue ART long-term because they feel well and do not have a clear understanding of the benefits of ART for health after delivery and/or breastfeeding. This misconception was described in a recent publication of perceptions of Option B+ in Malawi, in which women felt the program was being presented primarily for the benefit to the child (for prevention of MTCT) with less focus on concurrent benefits to the mother [13]. Women also reported confusion about Option B+ being presented as an "option" when there were no other therapeutic choices offered for PMTCT, and described feeling pressured to initiate ART immediately, with little or no support around decision-making for their health. There is a need to better understand the chal- 
lenges women face, and to develop evidence-based interventions to assist adherence and retention, particularly early in the continuum of care, such that the health benefits of Option B+ can be fully realized.

\section{Limitations}

Our study was limited by the number of available records at our three sites, with less availability of charts from the pre-Option B+ era as compared to the more recent Option B+ era, and as such, we may have had insufficient power to detect differences in default and adherence. The three facilities included in the study were selected due to specific affiliations with members of the research team, and may not be representative of other clinics and hospitals in Malawi based on regional differences or other site characteristics. This may explain the lower default rates than described elsewhere in Malawi and other similar settings. Because CD4 counts are not routinely done as part of Option $\mathrm{B}+$, information on CD4 count at ART initiation was available only for a small group of women in the study. Finally, we did not review data on infant outcomes, and included only maternal outcomes based on data available in the routine clinical charts (mastercards and registers). We therefore do not have information about the full spectrum of health issues faced by women and infants in each cohort, including opportunistic infections other than TB and KS and non-AIDS co-morbidities and events. We also do not have information on when default occurred in relation to delivery.

\section{Conclusion}

In our study sites, the transition to Option B+ was associated with ART initiation in healthier women and with lower mortality in the first year on ART. Although we did not find significant differences between default or adherence between cohorts, we saw trends toward these outcomes being more common among women in the Option B+ cohort. Taken together with emerging data from across Malawi, data suggest that further research is needed to better characterize adherence and default, including contributory factors. The success of Option $\mathrm{B}+$ will be determined by women's ability to remain on lifelong ART; therefore, characterizing challenges and designing interventions to support women will help ensure that the investment of resources in Option $\mathrm{B}+$ translates to long-term improvements in maternal and infant health.

\section{Author's Contributions}

AK, RH and LH, were involved with the design and conception of the study; AK, RH, LH, PJ and WW contributed to the intellectual content of the manuscript, WW conducted the data analysis. AK drafted the first manuscript and handled subsequent revisions with RH, LH, WW, and PJ.

\section{Acknowledgements}

We thank the patients, providers, and staff at Partners in Hope, Malamulo Hospital, and Thyolo District Hospital. We are grateful to John Hamilton and the Lilongwe-based EQUIP-Malawi staff for providing administration and oversight for this study.

\section{Funding}

This research has been supported by the President's Emergency Plan for AIDS Relief (PEPFAR) through USAID-Malawi under the terms of Grant No. 674-A-00-10-00035-00 and UCLA AIDS Institute, and the UCLA Center for AIDS Research (AI28697).

\section{Conflict of interest}

None.

\section{References}

[1] World Health Organization (2013) Consolidated Guidelines on the Use of Antiretroviral Drugs for Treating and Preventing HIV Infection: Recommendations for a Public Health Approach. World Health Organization, Geneva. http://www.who.int/hiv/pub/guidelines/arv2013/en/

[2] Government of Malawi (2008) Prevention of Mother to Child Transmission of HIV and Paediatric HIV Care Guide- 
lines. 2nd Edition, Ministry of Health, Lilongwe.

http://www.aidstar-one.com/sites/default/files/PMTCT\%20Guidelines\%20Malawi\%202008.pdf

[3] National Statistical Office (NSO)/ICF (2011) Malawi Demographic and Health Survey 2010. NSO and ICF Macro, Zomba, Malawi and Calverton. http://www.measuredhs.com/pubs/pdf/FR247/FR247.pdf

[4] Cooper, E.R., Charurat, M., Mofenson, L., Hanson, I.C., Pitt, J., Diaz, C., Hayani, K., Handelsman, E., Smeriglio, V., Hoff, R., Blattner, W. and Women and Infants’ Transmission Study Group (2002) Combination Antiretroviral Strategies for the Treatment of Pregnant HIV-Infected Women and Prevention of Perinatal HIV-1 Transmission. Journal of Acquired Immune Deficiency Syndromes, 29, 484-494. http://www.ncbi.nlm.nih.gov/pubmed/11981365 http://dx.doi.org/10.1097/00126334-200204150-00009

[5] Centers for Disease Control and Prevention (CDC) (2013) Impact of an Innovative Approach to Prevent Motherto-Child Transmission of HIV-Malawi, July 2011-September 2012. Morbidity and Mortality Weekly Report, 62, 148151. www.cdc.gov/mmwr/preview/mmwrhtml/mm6208a3.htm

[6] Tenthani, L., Haas, A.D., Tweya, H., Jahn, A., van Oosterhout, J.J., Chimbwandira, F., Chirwa, Z., Ng’ambi, W., Bakali, A., Phiri, S., Myer, L., Valeri, F., Zwahlen, M., Wandeler, G., Keiser, O. and Ministry of Health in Malawi and IeDEA Southern Africa (2014) Retention in Care under Universal Antiretroviral Therapy for HIV-Infected Pregnant and Breastfeeding Women (“Option B+") in Malawi. AIDS, 28, 589-598.

http://www.ncbi.nlm.nih.gov/pubmed/24468999

[7] Coulborn, R.M., Trivino Duran, L., Metcalf, C., Namala, Y., Chirwa, Z., Murowa, M., Mbewa, K. and Garone, D. (2013) Preliminary Findings of a Routine PMTCT Option B+ Program in a Rural District in Malawi. 7th IAS Conference on HIV Pathogenesis, Treatment and Prevention, Kuala Lumpur, 30 June-3 July 2013. http://pag.ias2013.org/Abstracts.aspx?AID=2280

[8] Ng'ambi, W.F., Tweya, H., Speight, C., Man-Bourdon, C., Hosseinpour, M. and Phiri, S. (2013) Determinants of Antiretroviral Treatment Adherence among Women Accessing PMTCT “Option B+”: A Retrospective Study at Bwaila Hospital, Malawi. 7th IAS Conference on HIV Pathogenesis, Treatment and Prevention, Kuala Lumpur, 30 June-3 July 2013. http://pag.ias2013.org/Abstracts.aspx?AID=2602

[9] Kaplan, R., Orrell, C., Zwane, E., Bekker, L.G. and Wood, R. (2008) Loss to Follow-Up and Mortality among Pregnant Women Referred to a Community Clinic for Antiretroviral Treatment. AIDS, 22, 1679-1681. http://www.ncbi.nlm.nih.gov/pmc/articles/PMC2741732/ http://dx.doi.org/10.1097/QAD.0b013e32830ebcee

[10] Kalembo, F.W. and Zgambo, M. (2012) Loss to Followup: A Major Challenge to Successful Implementation of Prevention of Mother-to-Child Transmission of HIV-1 Programs in Sub-Saharan Africa. ISRN AIDS, 2012, Article ID: 589817. http://www.ncbi.nlm.nih.gov/pmc/articles/PMC3767368/

[11] Wang, B., Losina, E., Stark, R., Munro, A., Walensky, R.P., Wilke, M., Martin, D., Lu, Z., Freedberg, K.A. and Wood, R. (2011) Loss to Follow-Up in a Community Clinic in South Africa-Roles of Gender, Pregnancy and CD4 Count. South African Medical Journal, 101, 253-257. http://www.ncbi.nlm.nih.gov/pmc/articles/PMC3834586/

[12] Nachega, J.B., Uthman, O.A., Anderson, J., Peltzer, K., Wampold, S., Cotton, M.F., Mills, E.J., Ho, Y.S., Stringer, J.S., McIntyre, J.A. and Mofenson, L.M. (2012) Adherence to Antiretroviral Therapy during and after Pregnancy in LowIncome, Middle-Income, and High-Income Countries: A Systematic Review and Meta-Analysis. AIDS, 26, $2039-2052$. http://www.ncbi.nlm.nih.gov/pubmed/22951634 http://dx.doi.org/10.1097/QAD.0b013e328359590f

[13] Rebekah Webb Consulting/ICW/GNP+ (2013) Understanding the Perspectives and/or Experiences of Women Living with HIV Regarding Option B+ in Uganda and Malawi. Global Network of People Living with HIV. http://www.gnpplus.net/assets/wbb_file_updown/2793/2013-Option-B+-Report-GNP-and-ICW.pdf 
Scientific Research Publishing (SCIRP) is one of the largest Open Access journal publishers. It is currently publishing more than 200 open access, online, peer-reviewed journals covering a wide range of academic disciplines. SCIRP serves the worldwide academic communities and contributes to the progress and application of science with its publication.

Other selected journals from SCIRP are listed as below. Submit your manuscript to us via either submit@scirp.org or Online Submission Portal.
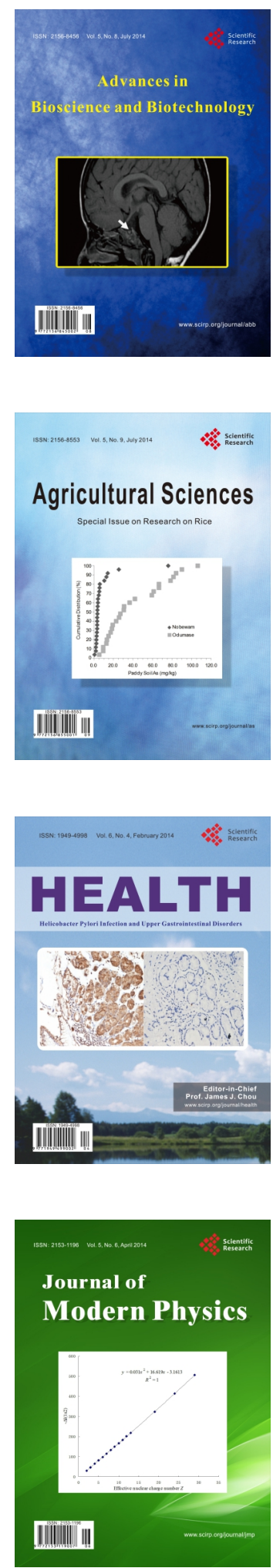
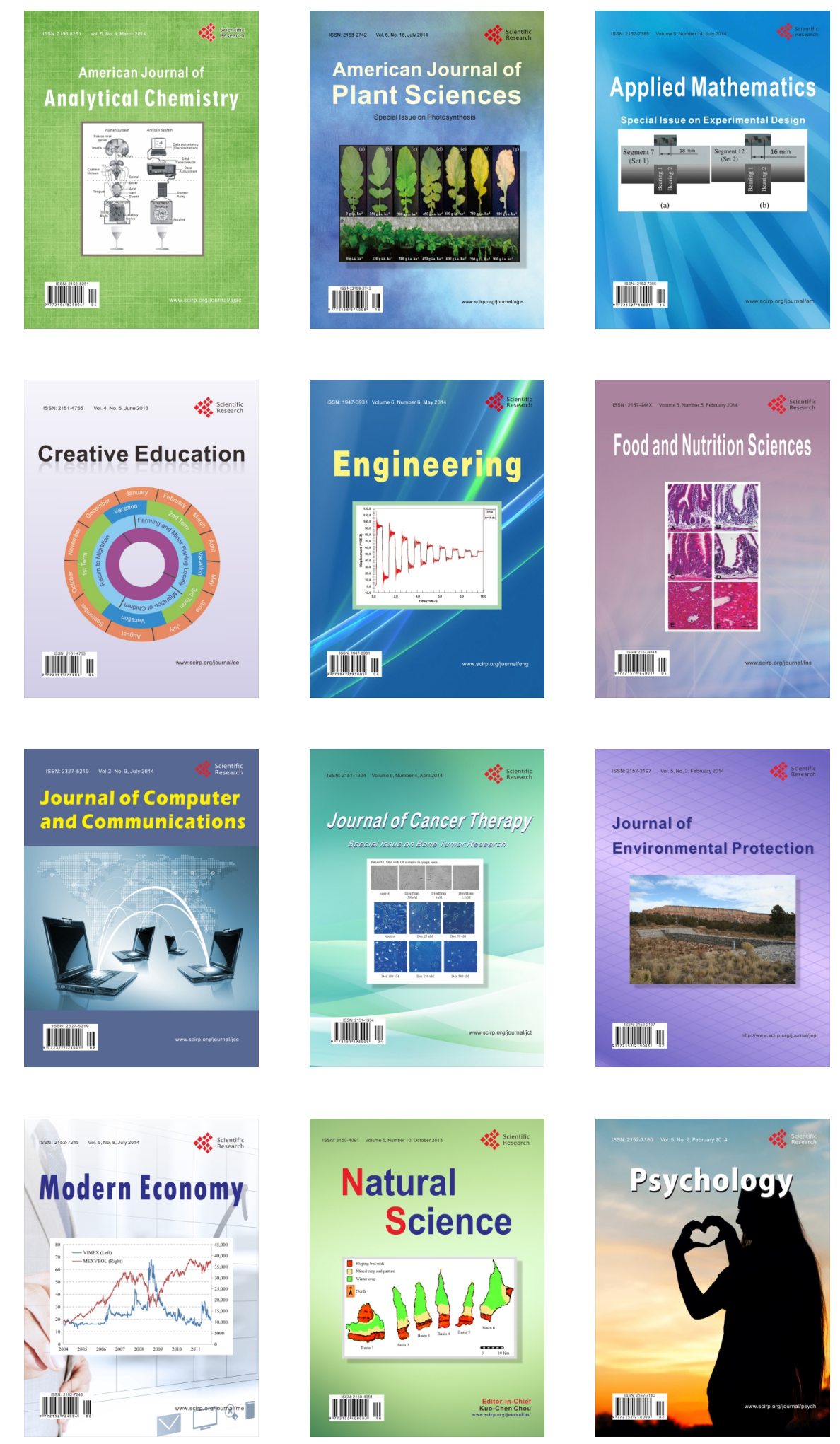